\title{
Ärzte sind keine Zielscheiben
}

\author{
Thomas Nierle \\ Dr. med., Präsident von Ärzte ohne Grenzen Schweiz
}

Stellen Sie sich vor, Sie arbeiten auf der Station oder sitzen in Ihrer Sprechstunde. Sie hören ein lautes Zischen und dann einen ohrenbetäubenden Knall. Die Wände wackeln, die Fenster zersplittern und Gegenstände fliegen wahllos durch den Raum. Aus Angst, Neugier, aber auch aus dem Bedürfnis, schnellstmöglich Hilfe zu leisten, rennen Sie hinaus und schauen, was passiert. Der Anblick, der sich Ihnen bietet, ist schauerlich: Die Notfallstation brennt und liegt teilweise in Trümmern - sie wurde gerade von einem Luftangriff getroffen. Sie hören Schreie, sehen menschliche Körperteile und viel Blut; unter den Opfern sind Menschen, die Sie gut kennen - Ihre Patienten, aber auch geschätzte Mitarbeiter und Freunde. Das Chaos ist unbeschreiblich trotzdem fühlen Sie sich gezwungen, das zu tun, was Sie am besten können: ärztliche Hilfe leisten.

Manche Leserin, mancher Leser wird sich nun fragen, welche Botschaft ich mit einem so realitätsfremden Beispiel vermitteln will. Realitätsfremd? Ganz und gar nicht, wenn Sie in Syrien, im Jemen oder im Südsudan Ihren ärztlichen Beruf ausüben. Spitäler in Kundus, Aleppo und kürzlich in Abs im Jemen sind nur ein
Bruchteil der von Ärzte ohne Grenzen geführten oder unterstützten Gesundheitseinrichtungen, die während des letzten Jahres bombardiert und zerstört wurden. Dabei kamen unzählige Patienten ums Leben und auch engagierte Ärzte und Pflegefachkräfte bezahlten mit ihrem Leben. Hinzu kommt, dass Bombardierungen aus der Luft nur ein kleiner Teil der Gewalt sind, denen Gesundheitseinrichtungen und deren Mitarbeiter heutzutage in Kriegsgebieten ausgesetzt sind. Exekutionen im Patientenbett, Schüsse, Plünderungen und Todesdrohungen schränken die medizinische Hilfe immer stärker ein - und damit auch den Raum für Menschlichkeit.

\section{Zivilbevölkerung am stärksten betroffen}

Aber letztendlich ist es die Zivilbevölkerung, die unter der Gewalt am meisten leidet. Wenn Spitäler nach einem Angriff geschlossen werden müssen, verlieren Hunderttausende von einem Tag auf den anderen den Zugang zur Gesundheitsversorgung. Menschen sterben an einfach behandelbaren Krankheiten oder bleiben

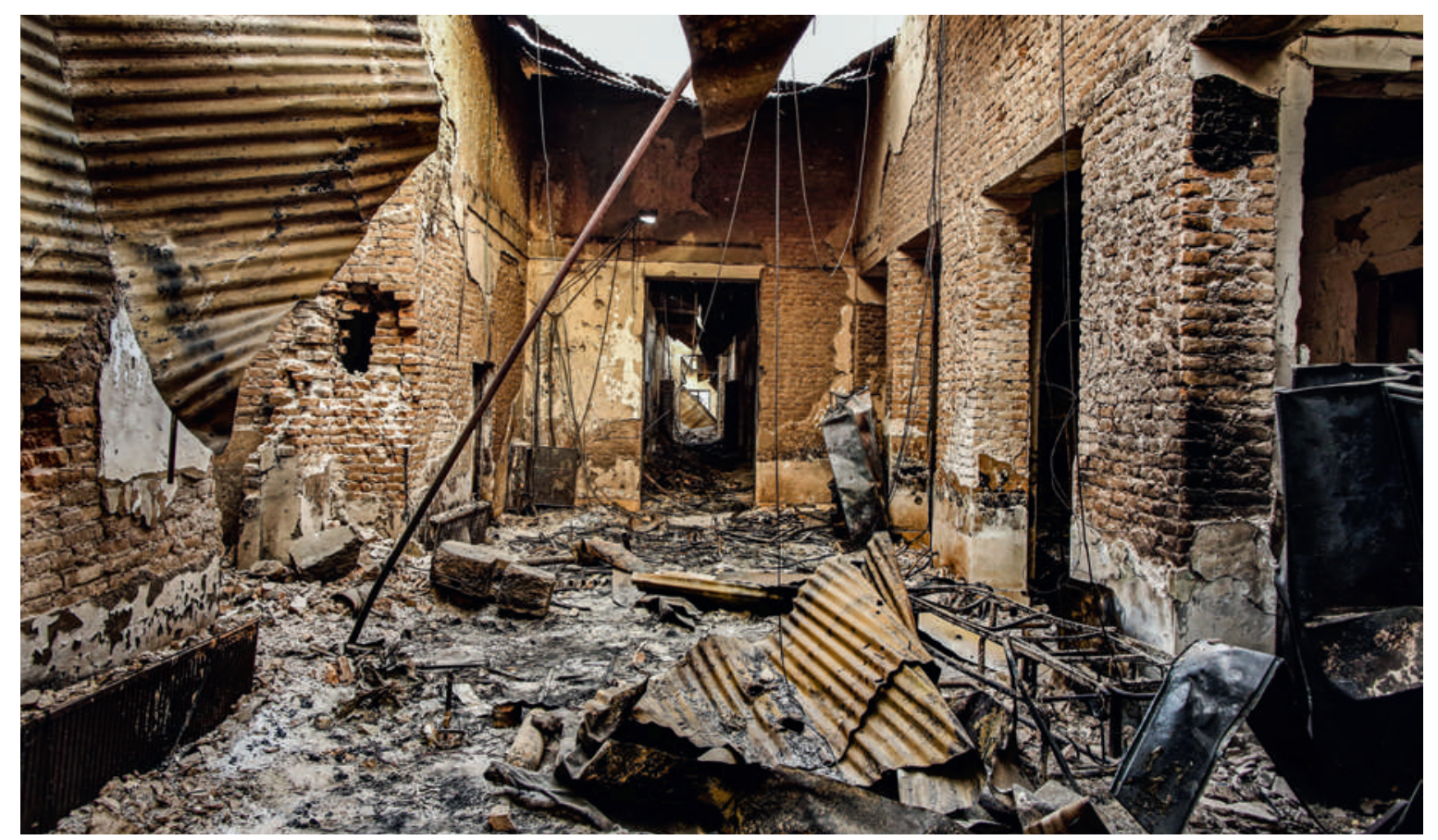

Im afghanischen Kundus ist das Traumatologie-Spital von MSF bei einem Luftangriff komplett zerstört worden. 42 Menschen starben, darunter 14 Mitarbeiter von MSF. 
ihr Leben lang verstümmelt, weil eine Verletzung nicht rechtzeitig versorgt werden konnte. Darüber hinaus ist und bleibt ein Spital ein Ort der Pflege, der menschlichen Aufmerksamkeit und der Hoffnung. Das Zerstören dieser Hoffnung scheint nun wieder Kriegstaktik geworden zu sein.

Eigentlich schützt das internationale humanitäre Völkerrecht Gesundheitseinrichtungen in Kriegszeiten. Weder Patienten noch medizinisches Personal dürfen angegriffen oder gar getötet werden. Nur hat sich die Auslegung des internationalen Völkerrechtes in Kriegen, in denen eine Kriegspartei systematisch als «Terroristengruppe» gebrandmarkt wird, radikal geändert. Den Kriegsparteien sind alle Mittel recht, um dem Gegner zusätzlichen Schaden zuzufügen. Dazu gehört, dafür zu sorgen, dass verwundete Kämpfer der Gegenpartei nicht behandelt werden, oder die Zivilbevölkerung so lange zu terrorisieren, bis sie keinen anderen Ausweg mehr sieht, als zu fliehen.

Terror wird mit Terror beantwortet und so entsteht eine Gewaltspirale, in der die Menschlichkeit auf dem Altar des totalen Krieges geopfert wird. Die Unparteilichkeit, welche fest in der medizinischen Ethik verankert ist, wird zur Zielscheibe! Denn wer verletzte Kämpfer behandelt, wird automatisch zu den Feinden gezählt.

\section{Solidarität als letztes Mittel}

Auch wenn die Staatengemeinschaft in einer UNO-Resolution im Mai diesen Jahres bestätigte, dass Spitäler und Gesundheitseinrichtungen sowie medizinisches und humanitäres Personal nicht angegriffen werden dürfen, so scheinen dies doch eher "fromme Wünsche» zu sein. Ob nun in Syrien oder im Jemen, diese Angriffe werden mit der Unterstützung von Grossmächten durchgeführt - Waffenlieferungen, logistische Unterstützung oder direkte Beteiligung an Kriegshandlungen machen unter anderen Russland, die Vereinigten Staaten und Grossbritannien mitverantwortlich. Die Kriegsparteien stempeln die Attacken auf Gesundheitseinrichtungen als Kollateralschäden ab aber kann man bei den 96 im Jahr 2015 bombardierten von Ärzte ohne Grenzen unterstützten Spitälern in Syrien noch von Zufall sprechen? Ist dies nicht eher eine systematische Zerstörung von Zivileinrichtungen, deren einziges Ziel die medizinische Versorgung der Bevölkerung ist?
Häufig werde ich gefragt, warum wir in diesen Regionen überhaupt noch versuchen weiterzuarbeiten. Wäre es nicht verantwortungsvoller gegenüber unseren Mitarbeitern, uns zurückzuziehen? Natürlich muss Ärzte ohne Grenzen seine Teams aus Gebieten evakuieren,

\section{\#NotATarget - \\ Veranstaltungen von MSF}

Mit einer Veranstaltungsreihe mit dem Titel "\#NotATarget» wird Médecins Sans Frontières / Ärzte ohne Grenzen (MSF) diesen Herbst Spitäler in der Schweiz besuchen. Zum Thema Angriffe auf medizinische Einrichtungen werden Ausstellungen gezeigt und es finden Abendanlässe mit Vorträgen, Filmvorführungen und Diskussionen statt.

HUG - Genfer Universitätsspitäler

Ausstellung vom 3. bis 9. Oktober 2016

Gedenkanlass am 3. Oktober ab 18.30 Uhr - ein Jahr nach

der Bombardierung des MSF-Spitals in Kundus, Afghanistan

CHUV - Universitätsspital Lausanne

Ausstellung vom 17. bis 23. Oktober 2016

Themenabend am 20. Oktober um $17 \mathrm{Uhr}$

Inselspital Bern

Ausstellung vom 17. bis 23. Oktober 2016

Themenabend am 18. Oktober um $18 \mathrm{Uhr}$

Hôpital Neuchâtelois - Neuenburger Spital

Ausstellung vom 24. bis 30. Oktober 2016

Kantonsspital Freiburg

Ausstellung vom 24. bis 30. Oktober 2016

Themenabend am 26. Oktober um $18 \mathrm{Uhr}$

Universitätsspital Basel

Ausstellung vom 31. Oktober bis 6. November 2016

Themenabend am 1. November um $18 \mathrm{Uhr}$

Tropeninstitut Basel

Ausstellung vom 7. bis 13. November 2016

Themenabend am 10. November um $18 \mathrm{Uhr}$

Kantonsspital Winterthur

Themenabend am 9. November 2016 um 18 Uhr

UniversitätsSpital Zürich

Ausstellung vom 14. bis 20. November 2016

Themenabend am 16. November um $18 \mathrm{Uhr}$

Weitere Informationen unter www.msf.ch/event

in denen Sicherheitsgarantien unzureichend sind oder Zwischenfälle ständig banalisiert werden. Es bleibt jedoch medizinisches Personal vor Ort zurück - lokale Ärzte und Pflegekräfte werden immer präsent sein, wo menschliches Leid durch medizinische Hilfe gelindert werden kann. Ein Rückzug von Ärzte ohne Grenzen ändert somit nichts an der Tatsache, dass Attacken gegen Gesundheitseinrichtungen aufhören müssen. Solidarität mit unseren Kollegen in den Kriegsgebieten ist eine Notwendigkeit, der wir uns nicht entziehen können, wenn wir noch daran glauben, dass Menschen in Kriegs- und Krisenzeiten ein Anrecht auf Schutz und medizinische Versorgung haben. Ärzte dürfen keine Zielscheiben sein!

Bildnachweis

(C) Andrew Quilty 